\section{Review: advice from doctors, counselling by nurses, behavioural interventions, nicotine replacement therapy, and several pharmacological treatments increase smoking cessation rates}

Lancaster T, Stead L, Silagy C, et al for the Cochrane Tobacco Addiction Review Group. Effectiveness of interventions to help people stop smoking: findings from the Cochrane Library. BMJ 2000 Aug 5;321:355-8.

\section{QUESTION: Are smoking cessation interventions effective?}

\section{Data sources}

Reviews were identified by searching the Cochrane Library.

\section{Study selection}

Reviews were selected if they included randomised controlled trials of interventions to reduce or prevent tobacco use that had $\geqslant 6$ months of follow up with outcomes of sustained abstinence or point prevalence quit rates.

\section{Data extraction}

\section{Main results}

20 systematic reviews were available in the Cochrane Library. 1 review (including 31 trials and $>26000$ participants who smoked) examined simple advice given by doctors during routine care and showed that the intervention increased quit rates (weighted odds ratio [OR] 1.69, 95\% CI 1.45 to 1.98). Another review of individual counselling given by nurses also showed increased quit rates.

Behavioural interventions for smoking cessation, in the forms of individual counselling (1 review) or group therapy (1 review), showed increased quit rates. The review of individual counselling (including 9 trials) showed that it was more effective than brief advice or usual care (weighted OR 1.55, CI 1.27 to 1.90). Group therapy was more effective than self help materials, but was not more effective than other interventions involving personal contact. 1 review of aversion therapy (including 24 trials), which paired the pleasurable stimulus of smoking with an unpleasant stimulus, found little evidence of beneficial effect. 1 review showed that self help interventions had no additional benefit over brief intervention (weighted OR 1.23, CI 1.02 to 1.49). A that personalised materials were more effective than standard materials (weighted OR 1.41 , CI 1.14 to 1.75 ).

A review of nicotine replacement therapy (including $>90$ trials) showed that all forms of this treatment were effective (weighted OR 1.71, CI 1.60 to 1.83). A review of anxiolytics and antidepressants showed that anxiolytics were not effective, whereas the antidepressants, bupropion and nortriptyline, increased quit rates. A review of the antihypertensive, clonidine (6 trials), showed effectiveness (weighted OR 1.89, CI 1.30 to 2.74) but limited
Extracted data included interventions and outcomes. personal advice, but were more effective than no comparison of self help interventions ( 8 trials) showed usefulness because of its side effect profile. Reviews of acupuncture, hypnotherapy, and exercise showed limited evidence of effectiveness for smoking cessation.

\section{Conclusion}

Advice from doctors, counselling by nurses, behavioural interventions (individual counselling or group therapy), nicotine replacement therapy, and several pharmacological interventions (such as the antidepressants, bupropion and nortriptyline) increase smoking cessation rates.
Sources of funding: $U K$ National Health Service Research and Development Programme Research Fund.

For correspondence: Dr T Lancaster, Imperial Cancer Research Fund General Practice Research Group, Department of Primar Health Care, University of Oxford, Institute of Health Sciences, Oxford OX3 7LF, UK. Fax +44 (0)1865227137.

A modified version of this abstract appears in Evidence-Based Medicine and Evidence-Based Mental Health. and the Imperial Cancer

\section{COMMENTARY}

The number of clinical trials evaluating the effectiveness of various approaches to smoking cessation reflects the strong interest of researchers and clinicians to find better ways to help individuals stop smoking. Lancaster et al's review of the 20 systematic reviews in the Cochrane Library on this topic is timely. An important strength of this report is that only Cochrane systematic reviews were included. Most systematic reviews done by Cochrane groups use a standard protocol and include a thorough search of published and unpublished studies. The results of this review are nevertheless dependent on the strengths and focuses of the individual systematic reviews that were included.

A systematic review by the US Department of Health and Human Services of $\geqslant 180$ clinical trials published between 1975 and 1999 also concluded that effective smoking cessation interventions exist that can produce long term or even permanent abstinence. ${ }^{1}$ Although the results of the US review are similar to conclusions reached in this review by Lancaster $e t a l$, some additional findings were reported. The US based group provided strong evidence that counselling intensity is associated with successful treatment outcomes. In addition, other efficacious strategies were identified such as telephone counselling. The panel that did the systematic review also developed clinical practice guidelines based on the available evidence.

Despite the high prevalence of smoking, healthcare providers often fail to assess or treat tobacco use consistently and effectively. ${ }^{2}$ Smoking status should be assessed for every patient. Because effective strategies are available, every patient who smokes should be offered at least a brief intervention in the form of a clear, strong statement of advice to stop smoking. For those willing to try to give up smoking, the use of practical counselling (problem solving or skills training) and pharmacotherapies (eg, nicotine replacement therapy) will increase the likelihood of success. It has been estimated that reducing current smoking by $50 \%$ would avoid about 175 million premature deaths worldwide. ${ }^{3}$ Delivery of effective cessation interventions should be a high priority.

Joan L Bottorff RN, PhD Professor and NHRDP Health Research Scholar School of Nursing, University of British Columbia Vancouver, British Columbia, Canada

1 Fiore MC, Bailey WC, Cohen SJ, et al. Treating tobacco use and dependence. A clinical practice guideline. Rockville, MD: US Department of Health and Human Services, 2000.

2 Jaen CR, Stange KC, Tumiel LM, et al. Missed opportunities for prevention: smoking cessation counseling and the competing demands of practice. J Fam Pract 1997;45:348-54.

3 Peto R, Lopez AD. The future worldwide health effects of current smoking patterns. In: Koop CE, Pearson CE, Schwarz MR, editors. Critical issues in global health. New York: Jossey-Bass (in press). 